\title{
Prediction of bending rigidity for laminated fabric with adhesive interlining by laminate model considered tensile and in-plane compressive moduli
}

\author{
KyoungOk Kim¹, Shigeru Inui ${ }^{2}$ and Masayuki Takatera ${ }^{2}$ \\ ${ }^{1}$ Department of Bioscience and Textile Technology, Interdisciplinary Division of Science and Technology, Shinshu
}

University, Japan

${ }^{2}$ Faculty of Textile Science and Technology, Shinshu University, Japan

\begin{abstract}
The purpose of this study is to predict bending rigidity of laminated fabric with adhesive interlining considered tensile and in-plane compressive moduli. The predicting method considering those moduli was proposed by theoretical derivation based on laminate model. Tensile and in-plane compressive moduli of neutral surface for face fabric and adhesive interlining respectively before laminating and modulus for bending rigidity were considered independently. The calculating equation for in-plane compressive modulus was proposed from the relationship between bending rigidities and tensile properties. The proposed predicting method was verified experimentally. Bending rigidities, tensile properties and thicknesses of adhesive interlinings, face fabrics and laminated fabrics with adhesive interlinings were measured by KES-FB system. The in-plane compressive moduli of adhesive interlinings were calculated by the proposed equation with the results of tensile properties for face fabrics. With the results of tensile and in-plane compressive moduli, the bending rigidities of laminated fabric with adhesive interlinings were calculated. The predicted bending rigidities considered with measured tensile properties and calculated in-plane compressive moduli were precisely closer to experimental results than the ones of the laminated model from our previous study. Therefore, this model gives a new way to predict bending rigidity of laminated fabric with adhesive interlining.
\end{abstract}

Keyword: bending rigidity, laminated fabric, woven fabric, adhesive interlining, tensile modulus, in-plane compressive modulus

\section{Introduction}

Fabrics for garments need variable properties for a suitable appearance and stability. Therefore, a large number of subsidiary materials have been used to fulfill those needs. In those materials, an adhesive interlining, which was used thermoplastic resin to bond, is a representative material to give clothing a suitable appearance and stability. However, the properties of woven fabric are considerably changed by laminating the adhesive interlining, and those property changes have been investigated by many researchers.

Namiranian et al. [1] investigated the plate buckling compression behavior of fused fabric composite using a specially designed clamp according to Dahlberg's test method. C. Cassidy et al. [2] studied the anisotropic mechanical behavior of woven fabrics, fusible interlinings and their composites in order to investigate the accuracy of equations used to predict the anisotropic linear elastic behavior of fabric for in-plane and bending deformation. Strazdiene et al. [3] investigated the method of punch deformation for the simulation of textile systems behavior and on the basis of that created an original method and found new criteria for shape stability evaluation. Fan et al. [4-6] suggested a set of equations to predict the low-stress mechanical properties of fused composites from those of composed fabric and fusible interlining fabrics. Fan and Leeuwner [7] also examined the causes of rippling, localized delamination or surface distortion in fused garments, theoretically and experimentally. Jeong et al. [8] reported on the construction of an integrated tool consisting of a neural network to select optimal interlining. Kanayama and Niwa [9, 10] suggested a prediction method for the bending rigidity of a composite based on mechanical theory for composite structures. Raj Sharma et al.11 investigated the effect of sewing and fusing of interlining on the drape behavior of men's suiting fabrics. Kim et al. [12] analyzed the suitability of non-woven fusible interlining to thin worsted fabric with various fabric structural parameters. Lai and Schenk [13] found the ideal composite condition range for interlining and face fabrics through discriminate analysis and the scatter plot. Shishoo et al. [14] introduced regression equations and investigated the relationship of mechanical properties theoretically and experimentally. Jevs nik et al. [15] analyzed some mechanical properties and parameters of drapability using different methods from two different points of the research area, using the finite element method. Jevšnik and Geršak [16] analyzed the dependence of the constructional parameters of fabrics on the properties of fused panels. Dawes and Owen [17] proposed a prediction method for the bending rigidity of a composite, which is considered tensile, and bending properties.

In the studies on adhesive interlining, the prediction method for bending rigidity was considered as one of the important themes. However, present studies about this point suggest the statically derived regression equation [5, 14] or the finite element method8 by relationships between theoretical results to experimental results for predicting bending rigidity, rather than using a theoretical base. Therefore, the propriety of those equations based on theoretical approach was insufficient.

On the other hand, Kanayama and Niwa [9, 10] and Dawes and Owen [17] suggested a prediction method of 
bending rigidity of the laminated composite with adhesive interlining based on laminate theory. However, the accuracy of those predicted results were not so high. Hence, the theoretical approach for the precise prediction method of the mechanical properties of laminated fabric with adhesive interlining, such as bending rigidity, was insufficient in those studies. Therefore, it is necessary to investigate the prediction method for bending rigidity with high accuracy from a theoretical point of view. Consequently, the method can be connected to the manufacturing and selection of more suitable adhesive interlining for the manufacturer and the customer.

In our previous study [18] prediction methods of bending rigidity for laminated fabric with adhesive interlining based on laminate theory were verified theoretically and experimentally. According to the verification, the prediction method for bending rigidity was proposed, considering the pressing effects on adhesive interlining and face fabric as well. The entire predicted results for bending rigidities from that method showed a better agreement with experimental ones than those results from existing prediction methods. However, for high accuracy of prediction, the necessity of an improvement for the new model was considered. Therefore, in this study, we proposed a new prediction method considering the elastic modulus for bending rigidity given by $E I$, where $E$ is elastic modulus and I is the moment of inertia of area, derived based on the laminate theory for ordinary solid materials. $E I$ values from the bending test are commonly used as bending rigidity. It is known that $E$ in $E I$ disagrees with the elastic modulus for the tensile modulus of a fabric. There are some studies about differences between those moduli. Osawa et al. [19] investigated the relationship between the extensional and in-plane compressive behavior of the fabrics using homogeneous plastic film, for which the Young's modulus is known, and showed the differences between extensional and bending moduli of the fabrics. Accordingly, the relationship between tensile moduli and bending moduli on a single fabric are independent.

In the case of a single fabric, no extension or compression on the neutral axis occurs while bending. However, while bending a laminated fabric, both composed fabrics separately undergo deformation, such as extension and compression, as shown in Figure 1. At the same time, neutral axes of both fabrics could be extended or compressed, respectively. Therefore, the extension or compression of neutral axes for both fabrics should be considered as a matter of bending rigidity for laminated fabric. Therefore, in this study, elastic moduli from tension and in-plane compression for each fabric before laminating and elastic moduli from bending rigidity were considered independently and a new prediction method for the bending rigidity of laminated fabric with adhesive interlining, considering tensile and in-plane compressive moduli, was proposed.

Dawes and Owen [17] suggested a similar idea for predicting bending rigidity, which considers the difference between tensile and bending properties. They used four types of face fabrics, adhesive interlinings and a paper to measure the apparent tensile and in-plane compressive moduli. They made the two types of composites, namely an adhesive interlining with a paper and a face fabric with a paper with a water-based polyvinyl alcohol adhesive. The tensile and in-plane compressive moduli were measured and calculated with those laminated fabrics. However, the predicted results did not agree with the experimental ones in their study, and the numbers of experimental samples were insufficient. Thus, in this study, we proposed an equation for calculating the in-plane compressive modulus, considering adhesive agent properties by theoretical derivation from the relationship between tensile and bending properties. From an experimental point of view, laminated fabrics with woven fabrics and adhesive interlinings were used for measuring those values. Consequently, the new prediction method for the bending rigidity of laminated fabric with adhesive interlining was verified with theoretical and experimental results.

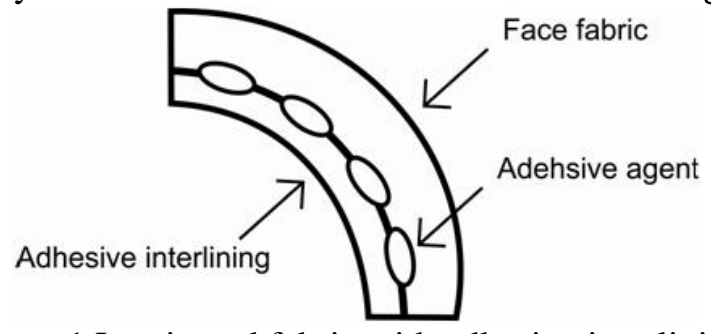

Figure 1 Laminated fabric with adhesive interlining.

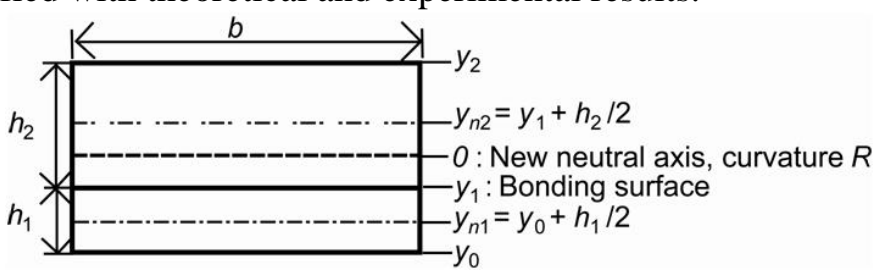

Figure 2 Structure model of laminated composite.

\section{Theoretical}

\section{Basic assumptions and structure model of laminated fabric}

Bending laminated fabric of two fabrics such as woven fabric and adhesive interlining, of which moduli are different, was investigated in this study. The adhesive and pressing effects for bonding those samples were considered already included by preparing pressed samples. The relationships between tensile and in-plane compressive moduli and bending deformation of laminated fabric with adhesive interlining were derived under the assumptions as follows for simplicity.

Firstly, neutral axes of face fabric and adhesive interlining before bonding pass through the centroid of those while bending respectively. Secondly, bending rigidities of face fabric and adhesive interlining are independent from tensile and in-plane compressive moduli of both fabrics at those centroids and tensile and in-plane compressive moduli are considered as constants. 
Structure model of laminated fabric with adhesive interlining, cloth ${ }_{1}$ and face fabric, cloth $_{2}$ is shown in Figure 2. $h_{1}$ and $h_{2}$ are the thickness of cloth ${ }_{1}$ and cloth 2 , respectively. $b$ is the breadth of cloths, $y_{n 1}$ and $y_{n 2}$ are the coordinates of the original neutral axis of cloth ${ }_{1}$ and cloth 2 , respectively, from the neutral surface in the cross section of the laminated fabric. $y_{0}$ and $y_{1}, y_{2}$ are the coordinates of surface and boundaries from the neutral surface in the cross section of the laminated fabric.

\section{Moment of face fabric, adhesive interlining and laminated fabric}

When the laminated fabric is bent, the strain distribution in the cross section is continuous. However the stress distribution is discontinuous at the boundary. The neutral surface is not consistent to the symmetry axis of the cross section. Stress of a laminated fabric can be express with the sum of stress from bending by neutral surface and that from strain by neutral surface.

From $\sigma=\varepsilon E=E \eta / R$, where $\sigma$ is the stress, $\varepsilon$ is the strain, $E$ is the modulus, $R$ is the radius of curvature of the neutral surface for the laminated fabric after bending and $\eta$ is the distance from the neutral surface in the laminated fabric, as assuming Bernoulli-Euler law, bending moment, $M_{1}$ of cloth ${ }_{1}$ is given by as follows.

$M_{1}=\int_{A_{1}} \eta \sigma d A_{1}=\int_{A_{1}} \frac{E}{R} \eta^{2} d A_{1}=\int_{y_{0}}^{y_{1}} \frac{E}{R} \eta^{2} b d \eta=\frac{E b}{R}\left[\frac{\eta^{3}}{3}\right]_{y_{0}}^{y_{1}}=\frac{E b}{R}\left\{\left[\frac{\eta^{3}}{3}\right]_{y_{0}}^{y_{n 1}}+\left[\frac{\eta^{3}}{3}\right]_{y_{n 1}}^{y_{1}}\right\}$

where $y_{n 1}=y_{0}+h_{1} / 2$.

On the other hand, we can divide the strain as follows.

$\varepsilon=\frac{\eta}{R}=\frac{y_{n 1}}{R}+\frac{\eta-y_{n 1}}{R}$

The first term in the right is the uniform strain over the cross section of cloth ${ }_{1}$ in the laminated fabric as bent. The second term is the strain from curvature, $R$ at the neutral plane cloth ${ }_{1}$ as bent alone.

When we set different elastic moduli for each strain as $E_{1 T}$, modulus for tension or compression of cloth ${ }_{1}$ and $E_{1 B}$, modulus for bending, the stress on the section corresponding to the strain is

$$
\sigma=\frac{y_{n 1}}{R} E_{1 T}+\frac{\eta-y_{n 1}}{R} E_{1 B}
$$

The moment of the stress is as follows.

$$
\begin{aligned}
M_{1} & =\int_{A 1} \eta \sigma d A_{1}=\int_{A_{1}} \eta\left(\frac{\eta-y_{n 1}}{R} E_{1 B}+\frac{y_{n 1}}{R} E_{1 T}\right) d A_{2} \\
& =\frac{E_{1 T}}{R} y_{n 1} \int_{A 1} \eta d A_{1}+\frac{E_{1 B}}{R} \int_{A 1}\left(\eta^{2}-\eta y_{n 1}\right) d A_{1}=\frac{E_{1 T}}{R} y_{n 1} \int_{y_{0}}^{y_{1}} \eta b d \eta+\frac{E_{1 B}}{R} \int_{y_{0}}^{y_{1}}\left(\eta^{2}-\eta y_{n 1}\right) b d \eta \\
& =\frac{E_{1 T}}{R} y_{n 1} b\left[\frac{\eta^{2}}{2}\right]_{y_{0}}^{y_{1}}+\frac{E_{1 B}}{R} b\left[\frac{\eta^{3}}{3}-y_{n 1} \frac{\eta^{2}}{2}\right]_{y_{0}}^{y_{1}}
\end{aligned}
$$

where $y_{0}=y_{n 1}-h_{1} / 2$ and $y_{1}=y_{n 1}+h_{1} / 2$, Equation (1.1) is as follows.

$$
M_{1}=\frac{1}{R}\left(E_{1 T} A_{1} y_{n 1}{ }^{2}+E_{1 B} I_{1}\right)
$$

Bending moment of cloth $2, M_{2}$ can be also expressed as follows.

$M_{2}=\frac{1}{R}\left(E_{2 T} A_{2} y_{n 2}{ }^{2}+E_{2 B} I_{2}\right)$

where $A_{1}=b h_{1}, A_{2}=b h_{2}$ and $I_{1}$ and $I_{2}$ are the moment of inertia of area for cloth ${ }_{1}$ and cloth ${ }_{2}$, respectively.

The first term in Equation (2) can be regarded as the bending moment by the uniform stress caused by tensile or compressive strain, of the neutral axes, $y_{n 1}$ during bending. The second term can be considered as bending moment by curvature, $R$. From the assumptions, the each modulus in Equation (2) were considered independently and those moduli were considered respectively as $E_{1 B}$, modulus for bending and $E_{1 T}$, modulus for tension or compression of cloth $_{1}$.

Similarly, for Equation (3), $E_{2 B}$ is the modulus for bending and $E_{2 T}$ is the modulus for tension or compression of cloth 2 . The case of $y_{n 1}>0$ means that the modulus is for the tensile strain and the case of $y_{n 1}<0$ means the modulus is for the compressive strain.

With those calculations, the bending moment of a laminated fabric, $M$ is given by

$M=M_{1}+M_{2}=\frac{1}{R}\left(E_{1 T} A_{1} y_{n 1}{ }^{2}+E_{2 T} A_{2} y_{n 2}{ }^{2}+E_{1 B} I_{1}+E_{2 B} I_{2}\right)$

In Equation (4), $E_{1 B} I_{1}$ and $E_{2 B} I_{2}$ are bending rigidities of cloth ${ }_{1}$ and cloth 2 , respectively, and the terms in parentheses refer to bending rigidity of laminated fabric. 


\section{Neutral surface of laminated fabric}

In equation (4), $y_{n 1}$ and $y_{n 2}$ are unknown. If the neutral axis of the laminated fabric is given, they can be obtained.

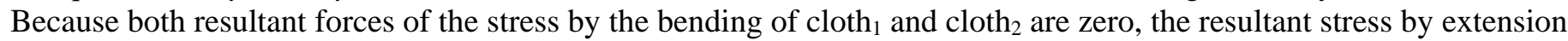
and compression of each neutral axis by the bending becomes zero. Then

$N=\frac{E_{1 T} y_{n 1} A_{1}}{R}+\frac{E_{2 T} y_{n 2} A_{2}}{R}=0$

where $y_{n 1} / R$ is the strain of the neutral surface for cloth ${ }_{1}$ and $y_{n 2} / R$ is the strain of the neutral surface for cloth ${ }_{2}$.

Therefore, we derive

$E_{1} h_{1} y_{n 1}+E_{2} h_{2} y_{n 2}=0$

Between $E_{I T}$ and $E_{2 T}$, if one is the modulus for tension, the other is the modulus for compression and the opposite case is also possible according to the direction of the curvature.

From $y_{n 2}=y_{1}+h_{2} / 2$ and $y_{n 1}=y_{0}+h_{1} / 2, \quad y_{1}=y_{0}+h_{1}=y_{n 1}+h_{1} / 2$ and $y_{2}=y_{1}+h_{2}$, we obtain

$y_{n 2}=y_{n 1}+\frac{\left(h_{1}+h_{2}\right)}{2}$

By substituting Equation (7) into (6), we obtain

$E_{1 T} h_{1} y_{n 1}+E_{2 T} h_{2} y_{n 1}+E_{2 T} h_{2} \frac{\left(h_{1}+h_{2}\right)}{2}=0$

Then,

$y_{n 1}\left(E_{1 T} h_{1}+E_{2 T} h_{2}\right)=-E_{2 T} h_{2} \frac{\left(h_{1}+h_{2}\right)}{2}$

Therefore, when $E_{1 T} h_{1}+E_{2 T} h_{2} \neq 0$, we obtain

$y_{n 1}=-\frac{E_{2 T} h_{2}\left(h_{1}+h_{2}\right)}{2\left(E_{1 T} h_{1}+E_{2 T} h_{2}\right)}$

In the same way, we obtain

$y_{n 2}=\frac{E_{1 T} h_{1}\left(h_{1}+h_{2}\right)}{2\left(E_{1 T} h_{1}+E_{2 T} h_{2}\right)}$

\section{Bending rigidity of laminated fabric}

With Equation (4) and (9), we can obtain moment of a laminated fabric as follows.

$M=\frac{1}{R}\left(E_{1 T} A_{1} y_{n 1}{ }^{2}+E_{2 T} A_{2}\left(y_{n 1}+\frac{h_{1}+h_{2}}{2}\right)^{2}+E_{1 B} I_{1}+E_{2 B} I_{2}\right)$

With Equation (10), we also derive

$y_{n 2}=y_{n 1}+\frac{h_{1}+h_{2}}{2}=\frac{E_{1 T} h_{1}\left(h_{1}+h_{2}\right)}{2\left(E_{1 T} h_{1}+E_{2 T} h_{2}\right)}$

and Equation (9) can be rewritten as follows by substituting Equation (11)

$M=\frac{1}{R}\left(\frac{b E_{1 T} h_{1} E_{2 T} h_{2}}{E_{2 T} h_{2}+E_{1 T} h_{1}}\left(\frac{h_{1}+h_{2}}{2}\right)^{2}+E_{1 B} I_{1}+E_{2 B} I_{2}\right)$

where

$\overline{E I}=\frac{b E_{1 T} h_{1} E_{2 T} h_{2}}{E_{2 T} h_{2}+E_{1 T} h_{1}}\left(\frac{h_{1}+h_{2}}{2}\right)^{2}+E_{1 B} I_{1}+E_{2 B} I_{2}$

If we express Equation (13) per breadth, and use $A_{1}=b h_{1}$ and $A_{2}=b h_{2}$, the bending rigidity of a laminated fabric per breadth is

$\frac{\overline{E I}}{b}=\frac{1}{b} \frac{E_{1 T} A_{1} E_{2 T} A_{2}}{E_{2 T} A_{2}+E_{1 T} A_{1}}\left(\frac{h_{1}+h_{2}}{2}\right)^{2}+\frac{E_{1 B} I_{1}}{b}+\frac{E_{2 B} I_{2}}{b}$

Bending rigidity per breadth can be measured by bending test such as KES-FB2 [14].

Calculating bending rigidity of laminated fabric

The bending rigidities, $B_{1}$ and $B_{2}$ of cloth ${ }_{1}$ and cloth ${ }_{2}$ can be expressed as per width.

$B_{1}=\frac{E_{1 B} I_{1}}{b}, \quad B_{2}=\frac{E_{2 B} I_{2}}{b}$ 
If the moment is proportional to the curvature, the measured bending rigidity by KES-FB2 can be used for these.

When we set $T_{1}$ and $T_{2}$ as apparent tensile modulus and apparent in-plane compressive modulus, those can be expressed as follows.

$T_{1}=\frac{E_{1 T} A_{1}}{b}, T_{2}=\frac{E_{2 T} A_{2}}{b}$

And the bending rigidity per unit breadth of a laminated fabric with adhesive interlining is

$\frac{\overline{E I}}{b}=B_{12}=\frac{T_{1} T_{2}}{T_{1}+T_{2}}\left(\frac{h_{1}+h_{2}}{2}\right)^{2}+B_{1}+B_{2}$

where $B_{12}$ is the bending rigidity of laminated fabric with adhesive interlining and face fabric.

The concept of Equation (17) was also proposed by Vivienne H. Dawes et al. [20]. In Equation (17), it is found that the bending rigidity of a laminated fabric with adhesive interlining is affected extremely by thickness of both cloths. In assumptions, we considered that $T_{1}$ and $T_{2}$ are linear function on bending rigidity if those are constant. In here, it should be noticed that $T_{1}$ is apparent tensile modulus and $T_{2}$ is apparent in-plane compressive modulus when cloth is $_{1}$ in outside and cloth ${ }_{2}$ is in inside of laminated fabric while bending. On the other hand, $T_{2}$ is apparent tensile modulus and $T_{1}$ is apparent in-plane compressive modulus in the opposite case.

In this equation, the apparent tensile modulus, $T_{1}$ can be obtained by a tensile tester. However the apparent in-plane compressive modulus, $T_{2}$ is hard to measure by a test especially in the case of fabric. Even though the apparent in-plane compressive modulus cannot be measured directly, $T_{1}, B_{1}, B_{2}, B_{12}, h_{1}$ and $h_{2}$ can be obtained experimentally. Therefore, from Equation (17), we can derive as follows.

$$
T_{2}=\frac{-T_{1}\left\{B_{12}-\left(B_{1}+B_{2}\right)\right\}}{B_{12}-\left(B_{1}+B_{2}\right)-T_{1}\left(\frac{h_{1}+h_{2}}{2}\right)^{2}}
$$

On the same calculations, we can obtain the opposite case of in-plane compressive modulus as follows.

$$
T_{1}=\frac{-T_{2}\left\{B_{12}-\left(B_{1}+B_{2}\right)\right\}}{B_{12}-\left(B_{1}+B_{2}\right)-T_{2}\left(\frac{h_{1}+h_{2}}{2}\right)^{2}}
$$

Therefore, $T_{2}$ can be obtained with Equation (18).

To confirm Equation (18), the tests with one kind of cloth $_{2}$, several kinds of cloth $_{1}$ and those laminated fabrics are needed to be carried out. In the case of laminated fabric with cloth ${ }_{1}$ at outside and cloth ${ }_{2}$ at inside, if the $T_{2}$ values of cloth ${ }_{2}$ in the cases of laminated fabrics with different cloth ${ }_{1}$ from Equation (18) are similar, the $T_{2}$ values are the apparent in-plane compressive modulus of cloth ${ }_{2}$. When $T_{2}$ values were obtained, the bending rigidities of different laminated fabrics can be predicted with Equation (17) with obtained $T_{2}$ values.

\section{Experimental}

To verify the proposed equations, four types of face fabric, 10 types of adhesive interlining and combined 40 types of laminated fabric bonded with adhesive interlining were prepared as samples and the bending rigidities, tensile properties and thicknesses were measured by the Kawabata Evaluation System (Katotech Co. KESFB). We controlled the weft density of five adhesive interlinings gradually increasing and we call those as CE-interlining. We also controlled the number of adhesive agent dots per area of five adhesive interlinings gradually increasing and we call those as DP-interlining. Bonding interlining to the face fabric was carried out by a press machine (KOBE DENKI KOGYOSYO, BP-V4812D) and the bonding condition was at $150^{\circ} \mathrm{C}$, under $29.4 \mathrm{kPa}$ load and for 10 s of pressing time.

Furthermore, it was clear from our previous study [18] that the pressing effect on the mechanical properties of laminate fabric after the laminating procedure needs to be considered. Therefore, pressed samples, which were pressed under the same bonding condition of laminating adhesive interlining with face fabric, were also prepared as samples in this study. Face fabric samples were pressed alone under the same condition of bonding interlining. For pressing adhesive interlining alone, polytetrafluoroethylene (PTFE) film (NITTO, No.900, $0.05 \mathrm{~mm} \times 300 \mathrm{~mm}$ ) was prepared for bonding the adhesive interlining. We bonded the adhesive interlining to PTFE film and removed the PTFE film from those composites. Those samples were called 'pressed face fabric' and 'pressed adhesive interlining', respectively. Specifications of those samples are shown in Tables 1 and 2. Every test was carried out under standard conditions (a temperature of $20 \pm 1^{\circ} \mathrm{C}$ and a relative humidity of $65 \pm 5 \%$ ). All samples were treated under standard conditions for 24 hours. Every test was conducted for five samples and the average was used. Bending properties of each sample were measured by the KES-FB2 pure bending tester [20]. The thickness of each sample was measured by the KES-FB3 compression tester at 49 Pa load. The tensile properties of each sample were measured by the KES-FB1 tensile tester until the maximum load of $490 \mathrm{cN} / \mathrm{cm}$. To determine the $T_{1}$ values of face fabric, it was necessary to consider the strain occurring during bending a fabric by KES-FB2. The curvature range of the bending rigidity by KES-FB2 is $0.5-1.5 \mathrm{~cm}^{-1}$. If the neutral surface, $\eta$, is determined as $0.025 \mathrm{~cm}$, which is assumed to be about 
one-quarter of the laminated fabric thicknesses, the strain range of a laminated fabric is $1.25 \%-3.75 \%$. Therefore, the T1values were obtained from the load at about $2.5 \%$ of the load-elongation curve, which is the elongation at curvature $1 \mathrm{~cm}^{-1}$, by a tensile tester, KES-FB1. With those obtained $T_{1}$ values, $T_{2}$ values were calculated by Equation (18), and the bending rigidities of laminated fabric with adhesive interlining were predicted by Equation (17).

Table 1 Specifications of face fabrics

\begin{tabular}{|c|c|c|c|c|c|c|}
\hline $\begin{array}{c}\text { Sample } \\
\text { name }\end{array}$ & Yarn Count(tex) & Weave & Width $(\mathrm{cm})$ & $\begin{array}{c}\text { Density }(/ \mathrm{cm}) \\
(\text { Warp } \times \text { Weft })\end{array}$ & Material & Pressed face fabric \\
\hline A & 16.5 tex $\times 2 ;$ R33tex & Twill & 148 & $28 \times 22$ & Wool $100 \%$ & P-A \\
\hline B & 14 tex $\times 2 ;$ R28tex & Twill & 148 & $29 \times 24$ & Wool 100\% & P-B \\
\hline C & 14 tex $\times 2 ;$ R28tex & Satin & 148 & $43 \times 29$ & Wool 85\%, Angora15\% & P-C \\
\hline D & 8.5 tex $\times 2 ;$ R17tex & Satin & 148 & $52 \times 35$ & Polyester $80 \%$, Wool 15\%, Cashmere 5\% & P-D \\
\hline
\end{tabular}

Table 2 Specifications of adhesive interlinings

\begin{tabular}{|c|c|c|c|c|c|c|c|c|}
\hline $\begin{array}{l}\text { Sample } \\
\text { name }\end{array}$ & $\begin{array}{l}\text { Width } \\
(\mathrm{cm})\end{array}$ & $\begin{array}{l}\text { Density } \\
(/ \mathrm{cm})\end{array}$ & $\begin{array}{c}\text { Adhesive } \\
\text { dot number } \\
(/ \mathrm{cm}) \\
(\text { warp } \times \text { weft })\end{array}$ & $\begin{array}{l}\text { Adhesive } \\
\text { dot size } \\
(\mathrm{mm})\end{array}$ & $\begin{array}{c}\text { Mass per unit } \\
\text { area } \\
\left(\mathrm{g} / \mathrm{m}^{2}\right)\end{array}$ & $\begin{array}{c}\text { Adhesive } \\
\text { Mass } \\
\text { without } \\
\text { Interlining } \\
\left(\mathrm{g} / \mathrm{m}^{2}\right) \\
\end{array}$ & $\begin{array}{l}\text { Screen } \\
\text { thickness } \\
(\mu \mathrm{m})\end{array}$ & $\begin{array}{l}\text { Pressed adhesive } \\
\text { interlining }\end{array}$ \\
\hline CE-1 & 96.5 & $38 \times 22$ & $10 \times 10$ & 0.17 & 36.2 & 8.6 & 200 & P-CE-1 \\
\hline CE-2 & 96.7 & $38 \times 23$ & $10 \times 10$ & 0.17 & 35.6 & 8.0 & 200 & P-CE-2 \\
\hline CE-3 & 97.0 & $38 \times 25$ & $10 \times 10$ & 0.17 & 36.5 & 8.3 & 200 & P-CE-3 \\
\hline $\mathrm{CE}-4$ & 97.5 & $37 \times 26$ & $10 \times 10$ & 0.17 & 36.5 & 8.1 & 200 & P-CE-4 \\
\hline CE-5 & 97.2 & $37 \times 26$ & $10 \times 10$ & 0.17 & $\begin{array}{l}35.7 \\
\end{array}$ & 7.7 & 200 & P-CE-5 \\
\hline DP-1 & 93.5 & $39 \times 24$ & $9 \times 9$ & 0.25 & 38.5 & 8.7 & 200 & P-DP-1 \\
\hline DP-2 & 93.5 & $39 \times 24$ & $10 \times 10$ & 0.23 & 39.9 & 10.0 & 150 & P-DP-2 \\
\hline DP-3 & 93.5 & $39 \times 24$ & $10 \times 10$ & 0.30 & 41.8 & 11.6 & 200 & P-DP-3 \\
\hline DP-4 & 93.5 & $39 \times 24$ & $11 \times 11$ & 0.20 & 37.5 & 8.7 & 200 & P-DP-4 \\
\hline DP-5 & 93.5 & $39 \times 24$ & $12 \times 12$ & 0.10 & 39.3 & 10.1 & 150 & P-DP-5 \\
\hline
\end{tabular}

\section{Results and discussion}

The difference between moduli from tensile and bending of face fabrics and adhesive interlinings

Before verifying Equation (17) and (18), the difference from $E_{b}$, a modulus for bending rigidity, and $E_{t}$, a modulus for tensile properties which was mentioned previously was investigated by comparing those moduli of prepared samples. It was shown by some research that the compressive modulus was lower than the tensile modulus [21]. The tensile strain by bending will be smaller than compressive strain. Therefore, the values of $E_{t}$ were obtained from the load at $2.5 \%$ of the load-elongation curve by tensile tester, KES-FB1. In this study, $B$ values of KES-FB2 on the case that a face fabric is outside while bending were used because an adhesive interlining are usually used inside of clothing in the field. The values of $E_{b}$ were obtained from $B$ values by KES-FB2, a pure bending tester. Woven fabrics and adhesive interlinings were considered as a rectangle.

Figure 3- 5 shows the difference of $E_{b}$ and $E_{t}$ of face fabrics and adhesive interlinings. The values of $E_{b}$ and $E_{t}$ were subsequently different and the values of $E_{t}$ were higher than those of $E_{b}$. The difference of between the tensile and bending modulus of adhesive interlining is larger than that of face fabrics. As a result, it was confirmed that the $E_{b}$ and $E_{t}$ are apparently different experimentally.

\section{Calculating $T_{1}$ and $T_{2}$ values of face fabrics and adhesive interlinings}

In this study, the pressed samples were used for measuring bending rigidity, thickness and tensile properties because it was found that the predicted results considered pressing effect were more agreed with experimental results in our previous study [18]. The measured bending rigidities and thicknesses of pressed samples are shown in Tables 3 and 4. In our previous study, the parameter changes of bending rigidity and thickness after pressing were considered. However, the changes of tensile properties were not considered. Therefore, the changes of tensile properties by pressing were also investigated in this study. Load-elongation curves of face fabrics before pressing and after pressing are shown in Figure 6. As shown in Figure 6, it was found that the elongation of face fabrics at the same load after pressing became larger than those before pressing. It will be necessary to investigate the reasons for elongation changing in the future.

In this study, the case where a face fabric is outside while bending is considered. Therefore, the apparent tensile modulus, $T_{1}$, of face fabrics and apparent in-plane compressive modulus, $T_{2}$, of adhesive interlinings were considered. Obtained $T_{1}$ values, calculated $T_{2}$ values and the average $T_{2}$ values of all samples are shown in Tables 5 and 6. The $T_{2}$ values of adhesive interlining from different face fabrics were close to each other. However, the calculated results were not exactly in agreement with each other, even though small differences were found, depending on samples. The reason could be the nonlinearity of fabric and adhesive permeation. The woven fabric has entirely non-linear tensile properties. However, the method to obtain $T_{1}$ values used the load at $2.5 \%$ elongation of the load-elongation curve and those curves do not show the non-linearity of woven fabric entirely. Because of the non-linearity of tensile properties for woven fabric, there would be some differences between $T_{2}$ values, depending on the types of face fabrics. Furthermore, the permeation of adhesive agent on the adhesive interlining could be another 
reason for differences. The permeation of adhesive agent was different depending on different types of face fabric, so the effects on mechanical properties by laminating adhesive interlining could be different. Among the calculated results, the results of $T_{2}$ for adhesive interlinings from face fabric $\mathrm{C}$ are slightly larger than those of other face fabrics, as shown in Table 6. The reason could be due to the assumptions that were mentioned for the first procedure. We assumed that the neutral axis of face fabric and adhesive interlining passes through the neutral axis of those while bending. However, depending on the structure and yarn properties of woven fabric, the placement of the neutral axis could be changed. In particular, face fabric $\mathrm{C}$ has the possibility not to pass through the centroid. It will be necessary to investigate this point in the future.

Including the differences of face fabric $\mathrm{C}$, the entire calculated results were similar each other. Therefore, it was possible to consider the $T_{2}$ values as apparent in-plane compressive moduli of adhesive interlinings.

Table 3 The bending rigidities and thicknesses of pressed interlinings

\begin{tabular}{|c|c|c|c|c|c|}
\hline Sample name & $\begin{array}{l}\text { Bending rigidity } \\
\left(\mathrm{cN} \cdot \mathrm{cm}^{2} / \mathrm{cm}\right)\end{array}$ & $\begin{array}{c}\text { Thickness } \\
(\mathrm{cm})\end{array}$ & Sample name & $\begin{array}{l}\text { Bending rigidity } \\
\left(\mathrm{cN} \cdot \mathrm{cm}^{2} / \mathrm{cm}\right)\end{array}$ & $\begin{array}{l}\text { Thickness } \\
(\mathrm{cm})\end{array}$ \\
\hline P-CE-1(warp) & 0.0058 & \multirow{2}{*}{0.0265} & P-DP-1(warp) & 0.0064 & \multirow{2}{*}{0.0235} \\
\hline P-CE-1(weft) & 0.0051 & & P-DP-1(weft) & 0.0024 & \\
\hline P-CE-2(warp) & 0.0058 & \multirow{2}{*}{0.0246} & P-DP-2(warp) & 0.0059 & \multirow{2}{*}{0.0239} \\
\hline P-CE-2(weft) & 0.0030 & & P-DP-2(weft) & 0.0020 & \\
\hline P-CE-3(warp) & 0.0060 & \multirow{2}{*}{0.0242} & P-DP-3(warp) & 0.0074 & \multirow{2}{*}{0.0245} \\
\hline P-CE-3(weft) & 0.0035 & & P-DP-3(weft) & 0.0033 & \\
\hline P-CE-4(warp) & 0.0070 & \multirow{2}{*}{0.0237} & P-DP-4(warp) & 0.0059 & \multirow{2}{*}{0.0239} \\
\hline P-CE-4(weft) & 0.0039 & & P-DP-4(weft) & 0.0027 & \\
\hline P-CE-5(warp) & 0.0085 & \multirow{2}{*}{0.0226} & P-DP-5(warp) & 0.0062 & \multirow{2}{*}{0.0247} \\
\hline P-CE-5(weft) & 0.0039 & & P-DP-5(weft) & 0.0013 & \\
\hline
\end{tabular}

Table 4 The bending rigidities and thicknesses of pressed face fabrics

\begin{tabular}{|c|c|c|}
\hline Sample name & $\begin{array}{l}\text { Bending rigidity } \\
\left(\mathrm{cN} \cdot \mathrm{cm}^{2} / \mathrm{cm}\right)\end{array}$ & Thickness(cm) \\
\hline P-A(warp) & 0.1353 & \multirow[b]{2}{*}{0.0516} \\
\hline P-A(weft) & 0.0758 & \\
\hline P-B(warp) & 0.0569 & \multirow[b]{2}{*}{0.0497} \\
\hline P-B(weft) & 0.0373 & \\
\hline P-C(warp) & 0.1389 & \multirow{2}{*}{0.0435} \\
\hline $\mathrm{P}-\mathrm{C}($ weft $)$ & 0.0647 & \\
\hline P-D(warp) & 0.0512 & \multirow[b]{2}{*}{0.0484} \\
\hline P-D(weft) & 0.0255 & \\
\hline
\end{tabular}

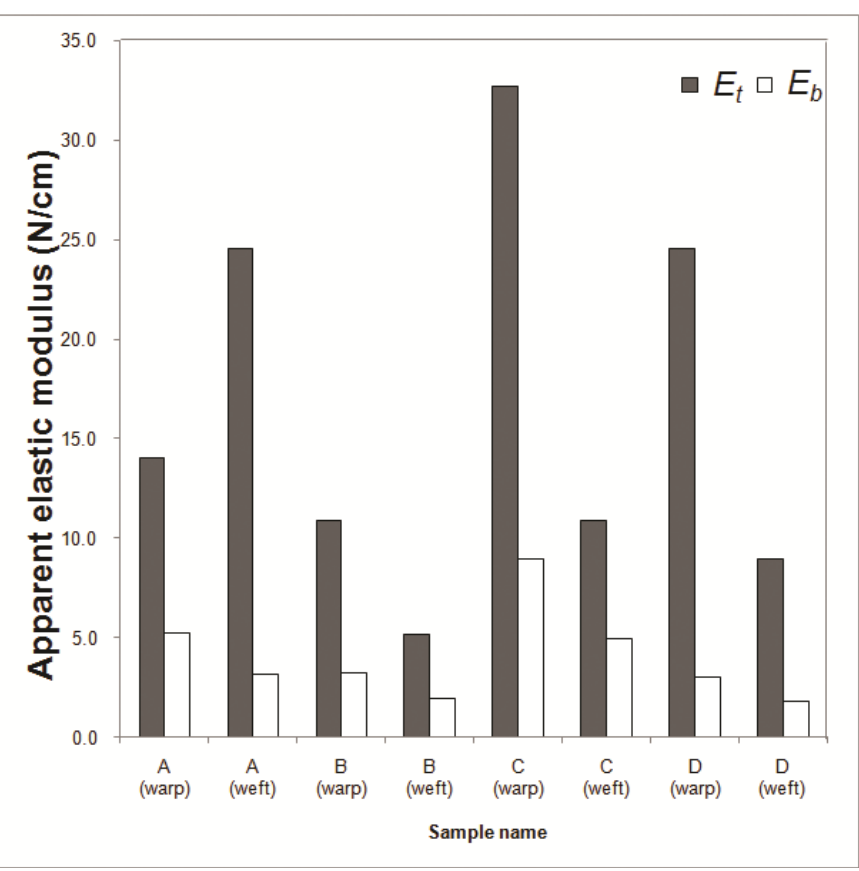

Figure 3 Comparison moduli from tension and bending of face fabric.
Table $5 T_{1}$ values of face fabrics

\begin{tabular}{|c|c|}
\hline Sample name & $T_{1}$ values $(\mathrm{N} / \mathrm{cm})$ \\
\hline A(warp) & 14.73 \\
\hline A(weft) & 29.47 \\
\hline B(warp) & 23.29 \\
\hline B(weft) & 4.33 \\
\hline C(warp) & 115.01 \\
\hline C(weft) & 13.31 \\
\hline D(warp) & 126.43 \\
\hline D(weft) & 23.29 \\
\hline
\end{tabular}

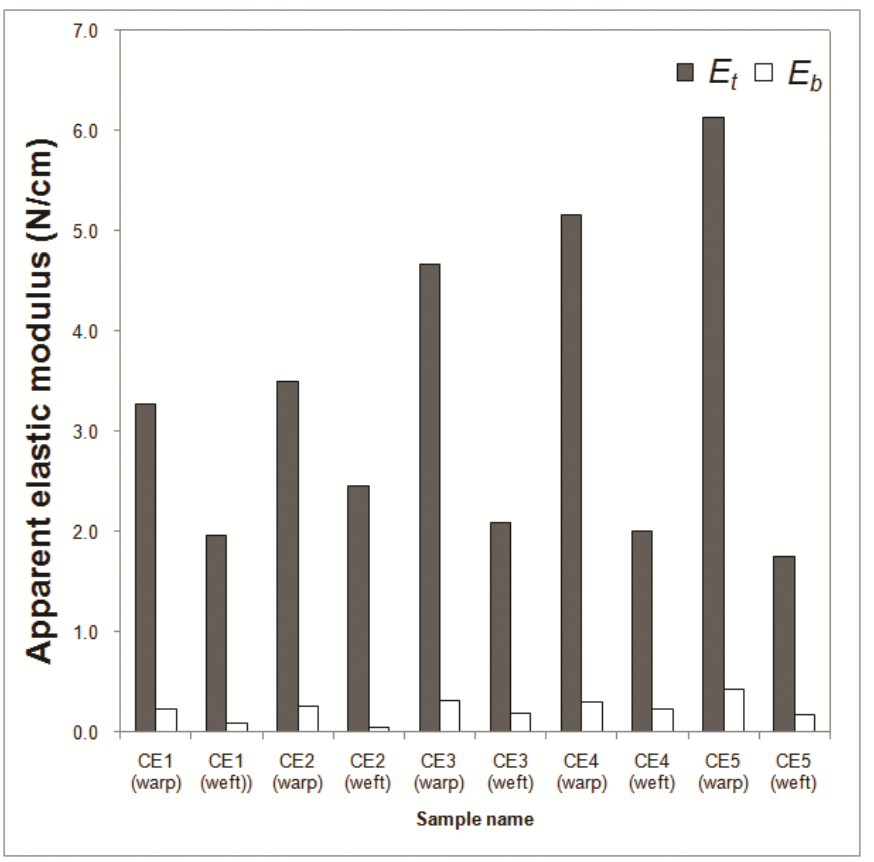

Figure 4 Comparison moduli from tension and bending of CE-interlining. 


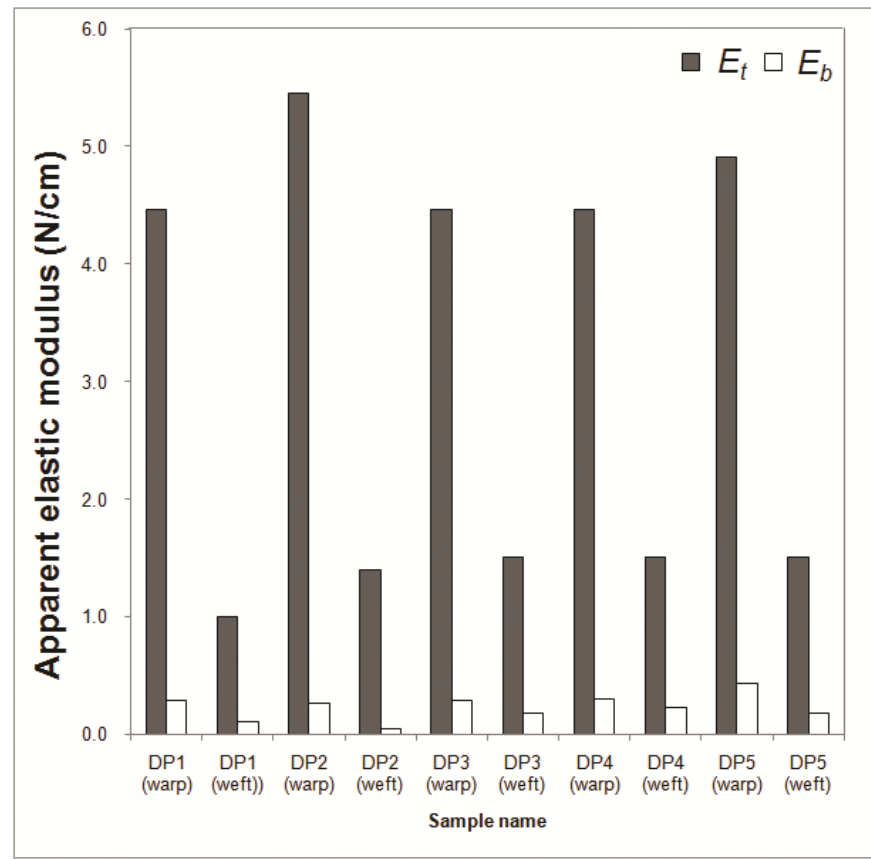

Figure 5 Comparison moduli from tension and bending of DP-interlining.

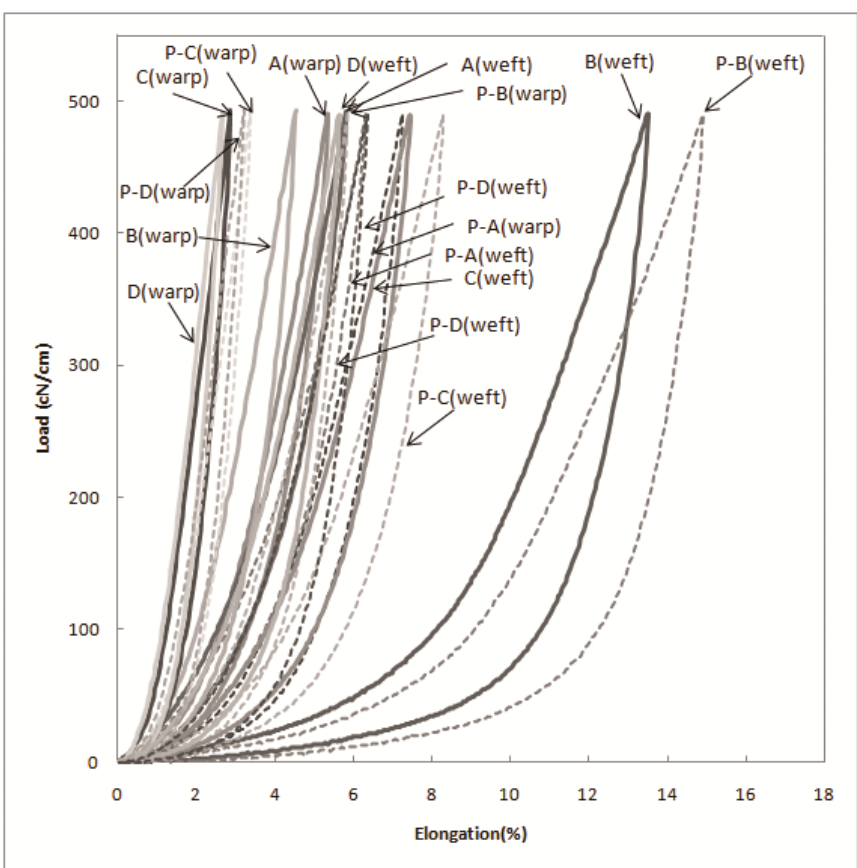

Figure 6 Load-Elongation curves of before pressing and after pressing for face fabrics

\section{Predicting the bending rigidities of laminated fabrics}

In this study, the averages of $T_{2}$ values for adhesive interlinings from all four face fabrics, as shown in Table 6 , were used for calculating bending rigidity to reduce experimental errors, depending on samples as mentioned previously. $T_{1}$ values of face fabric and the average $T_{2}$ values for adhesive interlining were used for verifying Equation (17) in order to predict the bending rigidity of laminated fabric with adhesive interlining. The predicted results with $T_{1}$ and the averages of $T_{2}$ values from parameters before pressing and after pressing are shown in Figures 7 and 8 . The absolute errors (AE-\%) and the mean absolute percentage error (MAPE-\%) for overall absolute errors (AE-\%) between experimental results and calculated ones of all samples are shown in Tables 7 and 8 . The entire predicted results were significantly close to the experimental results.

In particular, the results of weft direction for all samples were closer to the experimental ones than those of warp direction for all samples, as shown in Figure 7. The reason why the results in the weft direction were closer than the results in the warp direction was due to the $T_{1}$ values from tensile properties, which were less non-linear properties. The load-elongation curves of the weft direction showed linear properties between $0 \%$ and about $2.5 \%$ elongation, closer to the experimental results than those of warp direction.

The differences with predicted results of face fabric $\mathrm{C}$ were larger than those of the others, as shown in Figure 7. The reasons for some differences in the results of face fabric $C$ were considered to be the errors of the in-plane compressive moduli for face fabric C. Even though the predicted results for the tensile and in-plane compressive moduli with face fabric $\mathrm{C}$ showed some differences, the results still showed high agreement with this method compared to results from the laminate model. For the case of face fabric C, it will be necessary to be considered the precise reasons and another method to reduce the errors in the future.

Moreover, the predicted results with parameters of samples after pressing were closer to experimental results that those of samples after pressing, as shown in Figure 8. These were the same results as our previous study [18]. Pressing effects on the adhesive interlining and face fabric were found to be important factors to predict the bending rigidity of laminated fabric in our previous study. Elongation at $490 \mathrm{cN} / \mathrm{cm}$ load of face fabric after pressing became larger than that before pressing. It was also found that it was necessary to consider changes of tensile properties in the case of predicting bending rigidities for laminated fabric with adhesive interlining, considering tensile and in-plane compressive moduli.

Furthermore, these predicted results were compared with the ones from the laminated model of our previous study [18] as shown in Figure 9. Comparing those two results, the predicted results from tensile and in-plane compressive moduli were closer to the experimental ones than the ones with the laminated model. In our previous study [18] we controlled the weft density of CE-interlining, gradually increasing and bending rigidities in the warp direction of laminated fabrics, increasing with the density. However, this was not found in the predicted results in the previous study. On the other hand, the predicted results in this study increased with the density, as shown in Figure 7. Therefore, it was revealed that the prediction method, which considers the tensile and in-plane compressive moduli, is more suitable to reflect the effect of properties for adhesive interlinings and face fabric.

Consequently, the prediction method, considering the tensile and in-plane compressive moduli, is able to predict the bending rigidity of laminated fabric with adhesive interlining with high accuracy. 
Table $6 T_{2}$ values of pressed interlinings

\begin{tabular}{|c|c|c|c|c|c|}
\hline Sample name & $\begin{array}{c}T_{2} \text { values from } \\
\mathrm{A}(\mathrm{N} / \mathrm{cm})\end{array}$ & $\begin{array}{c}T_{2} \text { values from } \\
\mathrm{B}(\mathrm{N} / \mathrm{cm})\end{array}$ & $\begin{array}{c}T_{2} \text { values from } \\
\mathrm{C}(\mathrm{N} / \mathrm{cm})\end{array}$ & $\begin{array}{c}T_{2} \text { values from } \\
\mathrm{D}(\mathrm{N} / \mathrm{cm})\end{array}$ & $\begin{array}{c}\text { Average of } \\
T_{2} \text { values }(\mathrm{N} / \mathrm{cm})\end{array}$ \\
\hline CE-1(warp) & 0.70 & 0.68 & 0.92 & 0.62 & 0.73 \\
\hline CE-1(weft) & 0.34 & 0.37 & 0.31 & 0.28 & 0.33 \\
\hline CE-2(warp) & 0.81 & 0.68 & 1.06 & 0.75 & 0.82 \\
\hline CE-2(weft) & 0.39 & 0.36 & 0.39 & 0.32 & 0.37 \\
\hline CE-3(warp) & 0.87 & 0.63 & 1.06 & 0.80 & 0.84 \\
\hline CE-3(weft) & 0.41 & 0.38 & 0.40 & 0.31 & 0.37 \\
\hline CE-4(warp) & 0.93 & 0.78 & 1.15 & 0.90 & 0.94 \\
\hline CE-4(weft) & 0.41 & 0.39 & 0.40 & 0.33 & 0.38 \\
\hline CE-5(warp) & 1.06 & 0.89 & 1.24 & 1.06 & 1.06 \\
\hline CE-5(weft) & 0.40 & 0.42 & 0.42 & 0.35 & 0.40 \\
\hline DP-1(warp) & 1.29 & 1.08 & 1.47 & 1.05 & 1.23 \\
\hline DP-1(weft) & 0.31 & 0.32 & 0.36 & 0.28 & 0.32 \\
\hline DP-2(warp) & 1.39 & 0.10 & 1.58 & 1.09 & 1.26 \\
\hline DP-2(weft) & 0.37 & 0.34 & 0.59 & 0.30 & 0.40 \\
\hline DP-3(warp) & 1.48 & 1.02 & 1.67 & 1.17 & 1.33 \\
\hline DP-3(weft) & 0.35 & 0.35 & 0.44 & 0.30 & 0.36 \\
\hline DP-4(warp) & 1.45 & 1.06 & 1.58 & 1.15 & 1.31 \\
\hline DP-4(weft) & 0.33 & 0.32 & 0.38 & 0.30 & 0.33 \\
\hline DP-5(warp) & 1.32 & 0.99 & 1.54 & 1.07 & 1.24 \\
\hline DP-5(weft) & 0.33 & 0.30 & 0.33 & 0.28 & 0.31 \\
\hline
\end{tabular}

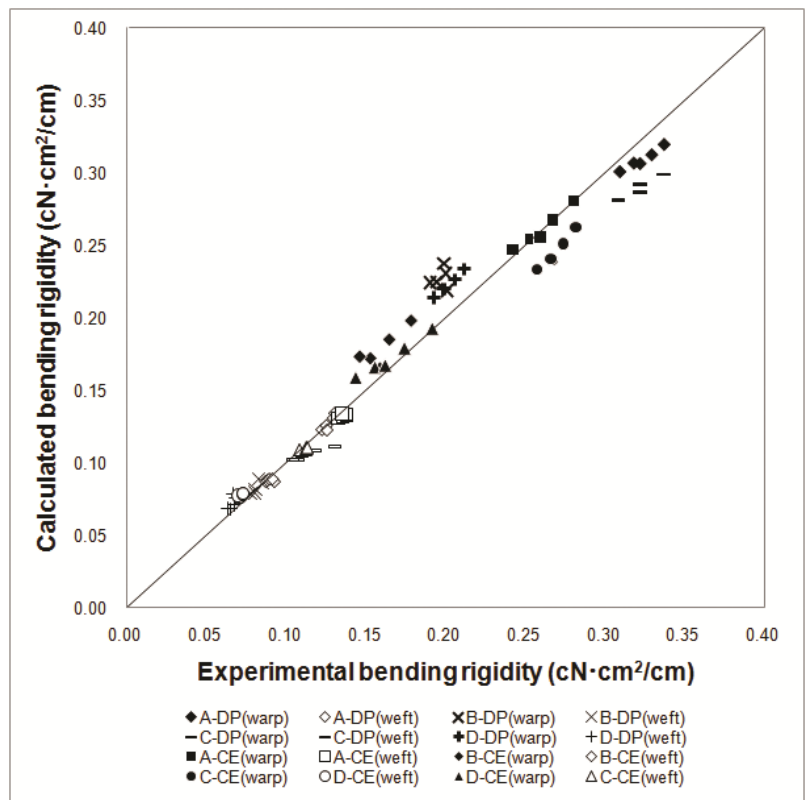

Figure 7 Comparison calculated bending rigidity and experimental bending rigidity with pressed samples.

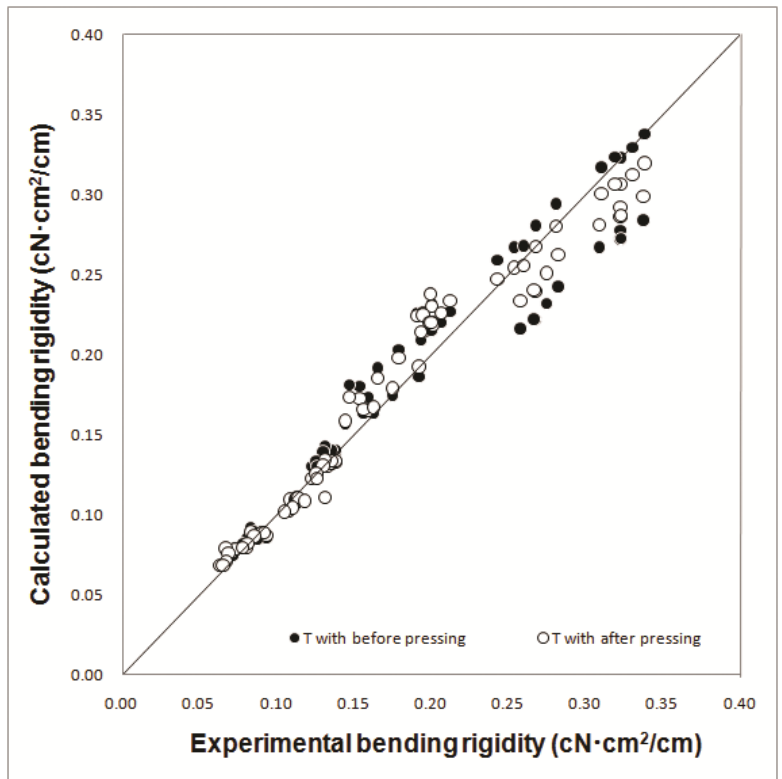

Figure 8 Comparison calculated bending rigidity and experimental bending rigidity with after pressing samples and before pressing samples.

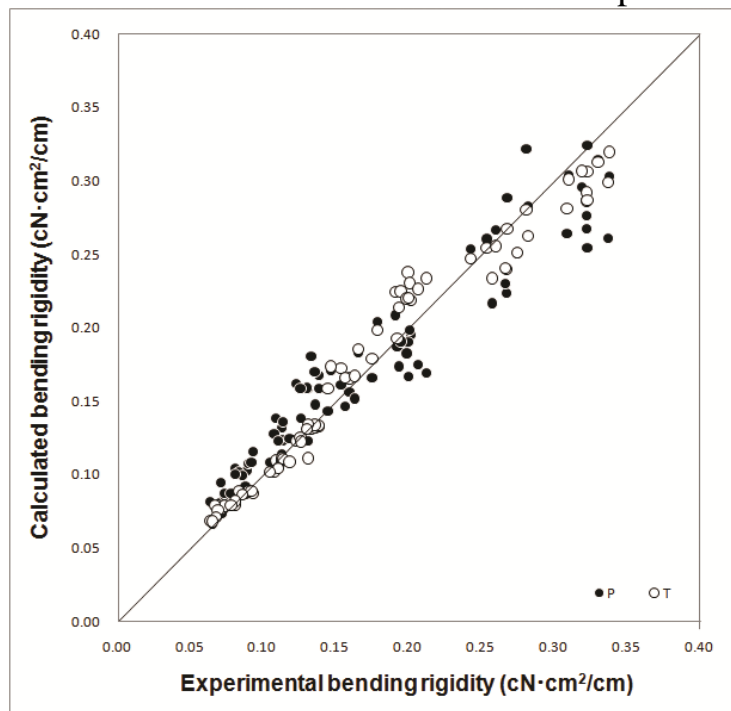

Figure 9 Comparison calculated bending rigidity and experimental bending rigidity of $\mathrm{P}$ and $\mathrm{T}$ methods (P: the method of laminate model with pressed samples, T: the method of tensile and in-plane compressive model with 


\section{Conclusion}

pressed samples)

A new prediction method for bending rigidity for laminated fabric with adhesive interlining, considering tensile and in-plane compressive moduli based on laminate theory, was proposed and verified experimentally. The apparent tensile modulus, $T_{1}$, and the apparent in-plane compressive modulus, $T_{2}$, were calculated and used to predict bending rigidities of laminated fabrics with adhesive interlinings. The obtained $T_{2}$ values of an adhesive interlining from the proposed equations were close to each other, even though the laminated fabric was made up of the different face fabrics. Therefore, the calculated values can be the in-plane compressive modulus of fabric. In the predicted bending rigidities, the entire predicted results were agreed with the experimental ones. Moreover, the results from the method considering tensile and in-plane compressive moduli were in better agreement with experimental results than the results of the laminate model. Even though some differences depending predicted results showed high accuracy. With these results, the prediction method, considering tensile and in-plane compressive moduli, is proposed as a prediction method of bending rigidity for laminated fabric with adhesive interlining and woven fabric with high accuracy. Moreover, the theoretical approach of this study will be applied to calculate in-plane compressive moduli for textiles, which cannot be measured experimentally.

Table 7 Absolute error (\%) between experimental results and predicted ones of each sample

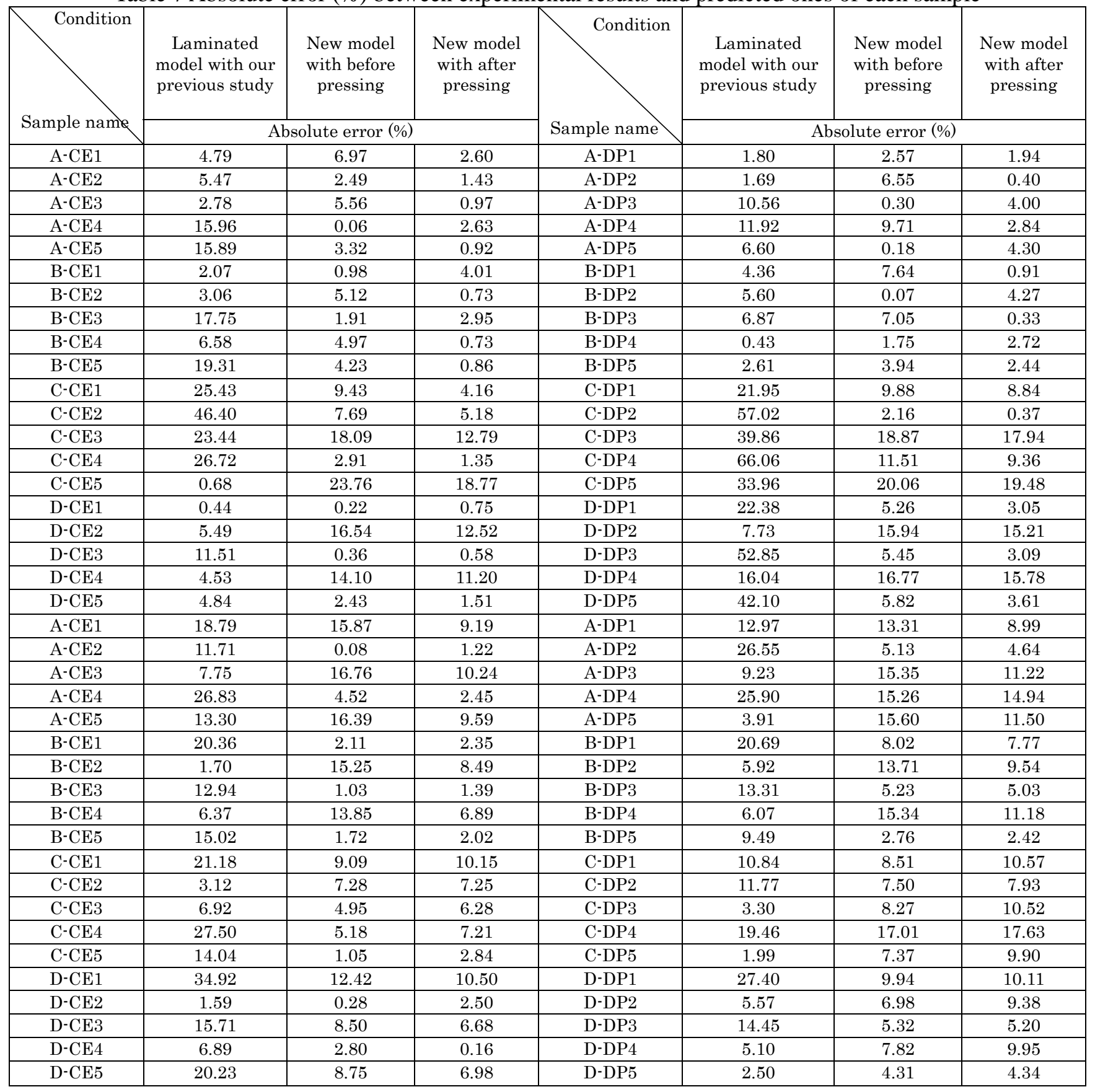

Table 8 Mean absolute percentage error (MAPE-\%) for aver all absolute errors (\%) of all samples Mean absolute percentage error (MAPE-\%) for aver all absolute errors (\%) of all samples 


\section{Acknowledgements}

The authors would like to express their appreciation to NITTOBOSEKI CO., LTD for providing the experimental samples.

\section{Funding}

This work was supported by Grant-in-Aid for Global COE Program by the Ministry of Education, Culture, Sports, Science, and Technology of Japan.

\section{References}

1. B. Namiranian, S. Shaikhzadeh Najar, A. Salehzadeh Nobari, "Compression plate buckling behavior of fused fabric composites”, International Journal of Clothing Science and Technology, Vol.21, No.5, pp.311-325 (2009)

2. C. Cassidy and S. V. Lomov, "Anisotropy of fabrics and fusible interlinings”, International Journal of Clothing Science and Technology, Vol.10, No.5, pp.379-330 (1998)

3. Eugenija Strazdiene, Matas Gutauskas, “The evaluation of fused knitted systems stability”, International Journal of Clothing Science and Technology, Vol.15, No.3/4, pp.204-210 (2003)

4. J. Fan, W. Leeuwner, and L. Hunter ; "Compatibility of Outer and Fusible Interlining Fabrics in Tailored Garments, part 1 : Desirable range of mechanical properties of fused composites”, Textile Research Journal, Vol. 67(2), 137-142 (1997)

5. J. Fan, W. Leeuwner, and L. Hunter, "Compatibility of Outer and Fusible Interlining Fabrics in Tailored Garments part 2 : Relationship between mechanical properties of fused composites and those of outer and fusible interlining fabrics”, Textile Research Journal, Vol. 67, No. 3, 194-197 (1997)

6. J. Fan, W. Leeuwner, and L. Hunter, "Compatibility of Outer and Fusible Interlining Fabrics in Tailored Garments part 2 :Selecting fusible interlinings”, Textile Research Journal, Vol. 67, No. 4, pp. 258-262 (1997)

7. J. Fan, W. Leeuwner, "The causes and prevention of rippling or localized delamination in fused garment parts", International Journal of Clothing Science and Technology, Vol.9, No.3, pp.228-235 (2005)

8. Sung H. Jeong, Jung H. Kim and Cheol J. Hong ; "Selecting Optimal Interlinings with a neural Network", Textile Research Journal, pp.1005, (2000)

9. Kanayama M and Niwa M. Bending properties of composite fabrics reinforced with fusible interlinings. J. Textile Machinery Soc Japan 1981; 35(1): 102-112.

10. Kanayama M and Niwa M. Theoretical calculation of bending rigidity of fused fabric composite. $J$. Textile Machinery Soc Japan 1984; 37(10): T161-T167.

11. Kaushal Raj Sharma, B.K. Behera, H. Roedel, Andrea Schenk, "Effect of sewing and fusing of interlining on drape behaviour of suiting fabrics", International Journal of Clothing Science and Technology, Vol.17, No.2, pp.75-90 (2005).

12. S.J. Kim, K.H. Kim, D.H. Lee, G.H. Bae, "Suitability of nonwoven fusible interlining to the thin worsted fabrics", International Journal of Clothing Science and Technology, Vol.10, No.3/4, pp.273-282 (1998)

13. Sang-Song Lai, Andrea Schenk, "Optimal combinations of face and fusible interlining fabrics", International Journal of Clothing Science and Technology Vol.13, No.5, pp.322-338 (2001)

14. Roshan Shishoo, Per Henrik Klevermar, Margareta Cednas, and Bertul Olofsson; "Multilayer Textile Structure", Textile Research Journal, pp. 669-679 (1971)

15. Simona Jevšnik, Jelka Geršak, Ivan Gubenšek, "The advance engineering methods to plan the behaviour of fused panel”, International Journal of Clothing Science and Technology, Vol.17, No.3/4, pp.273-282 (2005)

16. Simona Jevšnik, Jelka Geršak, "Use of a knowledge base for studying the correlation between the constructional parameters of fabrics and properties of a fused panel”, International Journal of Clothing Science and Technology, Vol.13, No.3/4, pp.186-197 (2001)

17. Vivienne H. Dawes and J. D. Owen, "The handle and bending behavior of fabric laminates", J.Text. Inst., 63, T443 (1972).

18. KyoungOk Kim, Shigeru Inui, and Masayuki Takatera, Textile Research Journal, Verification of Prediction for Bending Rigidity of Woven Fabric Laminated with Interlining by Adhesive Bonding, Vol. 81, Issue 6, pp. 598-607(2011).

19. Tadashi Osawa, Akira Nakayama, Kazuno Mihira, "On the relationship between the extensional and compressional behavior of the fabrics”, SEN-I GAKKAISHI, Vol. 29, No. 9, pp.T363-T367(1973)

20. S. Kawabata ; “The standardization and analysis of hand evaluation”, $2^{\text {nd }}$ ed., Textile Machinery society of Japan (1980)

21. Mituo Nakura and Mayumi Mizutani, On the position of the netral plane in the woven fabrics to which the pure bending applied, Journal of the Japan Research Association for Textile End-Use, Vol.29, pp.193-198(1988) 